\title{
First-principles study of defects and adatoms in silicon carbide honeycomb structures
}

\author{
E. Bekaroglu, ${ }^{1}$ M. Topsakal, ${ }^{1}$ S. Cahangirov, ${ }^{1}$ and S. Ciraci ${ }^{1,2, *}$ \\ ${ }^{1}$ UNAM-Institute of Materials Science and Nanotechnology, Bilkent University, Ankara 06800, Turkey \\ ${ }^{2}$ Department of Physics, Bilkent University, Ankara 06800, Turkey \\ (Received 3 December 2009; revised manuscript received 1 February 2010; published 24 February 2010)
}

\begin{abstract}
We present a study of mechanical, electronic and magnetic properties of two-dimensional (2D), monolayer of silicon carbide $(\mathrm{SiC})$ in honeycomb structure and its quasi-one-dimensional (quasi-1D) armchair nanoribbons using first-principles plane-wave method. In order to reveal dimensionality effects, a brief study of three-dimensional (3D) bulk and 1D atomic chain of SiC are also included. Calculated bond-lengths, cohesive energies, charge transfers and band gaps display a clear dimensionality effect. The stability analysis based on the calculation of phonon frequencies indicates that $2 \mathrm{D} \mathrm{SiC} \mathrm{monolayer} \mathrm{is} \mathrm{stable} \mathrm{in} \mathrm{planar} \mathrm{geometry.} \mathrm{We} \mathrm{found}$ that $2 \mathrm{D} \mathrm{SiC} \mathrm{monolayer} \mathrm{in} \mathrm{honeycomb} \mathrm{structure} \mathrm{and} \mathrm{its} \mathrm{bare} \mathrm{and} \mathrm{hydrogen} \mathrm{passivated} \mathrm{nanoribbons} \mathrm{are} \mathrm{ionic,}$ nonmagnetic, wide band gap semiconductors. The band gap is further increased upon self-energy corrections. The mechanical properties are investigated using the strain energy calculations. The effect of various vacancy defects, adatoms, and substitutional impurities on electronic and magnetic properties in 2D SiC monolayer and in its armchair nanoribbons is also investigated. Some of these vacancy defects and impurities, which are found to influence physical properties and attain magnetic moments, can be used to functionalize SiC honeycomb structures.
\end{abstract}

DOI: 10.1103/PhysRevB.81.075433

PACS number(s): 73.22.-f, 75.75.-c, 63.22.-m

\section{INTRODUCTION}

Owing to its exceptional thermal and physical properties, ${ }^{1}$ silicon carbide $(\mathrm{SiC})$ is a material, which is convenient for high temperature and high power device applications. Because of its wide band gap, $\mathrm{SiC}$ bulk structure has been a subject of active study in optical and optoelectronic research. Unlike the polymorphs of carbon, $\mathrm{SiC}$ is a polar material. In spite of the fact that both constituents of SiC are Group IV elements, charge is transferred from $\mathrm{Si}$ to $\mathrm{C}$ due to higher electronegativity of $\mathrm{C}$ relative to $\mathrm{Si}$ atom.

Bulk $\mathrm{SiC}$ has six commonly used stacking configurations denoted as $3 \mathrm{C}$ (zincblende), $2 \mathrm{H}$ (wurtzite), 4H, 6H, 15R, and 21R. Lubinsky et $\mathrm{al}^{2}{ }^{2}$ reported optical data related with indirect transitions, dielectric function and reflectivity of $3 \mathrm{C} \mathrm{SiC}$ using first-principles Hartree-Fock-Slater method. A more comprehensive study ${ }^{3}$ using orthogonalized linear combination of atomic orbitals (OLCAOs) method comprises the calculations of lattice constants, electronic band structure and optical properties of all six stacking configuration of $\mathrm{SiC}$.

As for $\mathrm{SiC}$ in lower dimensionality, $\mathrm{SiO}_{2}$ coated $\mathrm{SiC}$ nanowires ${ }^{4}$ were synthesized and showed favorable photocatalytic behavior. A theoretical work on hydrogen passivated $\mathrm{SiC}$ nanowires ${ }^{5}$ provided the energy bands both using local density approximation within density functional theory (DFT) and $s p^{3} s^{*}$ LCAO tight binding (TB) methods. SiC$\mathrm{ZnS}$ core-shell structures were also fabricated. ${ }^{6}$ Zincblende $\mathrm{SiC}$ nanoparticles were synthesized by carbothermal reduction method. ${ }^{7}$ Band gap of zincblende nanoparticles were estimated to be around $3 \mathrm{eV}$ from photoluminescence measurements. With a similar carbothermal method, microribbons ${ }^{8}$ with widths in the range of $500 \mathrm{~nm}-5 \mu \mathrm{m}$ and thickness of 50-500 nm were synthesized.

$\mathrm{SiC}$ is frequently used as a substrate to grow other materials. ${ }^{9,10} \mathrm{Few}$ layers of graphene was also grown on $\mathrm{SiC} .{ }^{11} \mathrm{SiC}$ clusters $\left(\mathrm{Si}_{n} \mathrm{C}_{n}, n=1-10\right)$ were investigated ${ }^{12}$ us- ing DFT. With the aim of developing a material for future nanoelectronic applications, binding energy, HOMO-LUMO gap, Mulliken charge, vibrational spectrum and ionization potential of $\mathrm{Si}_{n} \mathrm{C}_{n}$ clusters are revealed.

Earlier, planar honeycomb structure of graphite was exfoliated and its physical properties were analyzed. ${ }^{13-15}$ While graphene is a strictly planar crystal, the planar honeycomb structure of $\mathrm{Si}$ is unstable, but it is stabilized through puckering. ${ }^{16}$ Since the honeycomb structure is common to both $\mathrm{C}$ and $\mathrm{Si}$, one expects that stable $2 \mathrm{D} \mathrm{SiC}$ in honeycomb structure can be synthesized.

In this paper, a comprehensive analysis of the atomic, electronic and magnetic properties of two-dimensional (2D) monolayer of $\mathrm{SiC}$ honeycomb structure and its bare and hydrogen passivated armchair nanoribbons (A-SiCNR) are carried out using first-principles calculations. In spite of the fact that $2 \mathrm{D} \mathrm{SiC}$ monolayer is not synthesized yet, this study demonstrates its stability based on reliable theoretical methods. Furthermore, various mechanical, electronic, and magnetic properties are revealed. We started with the discussion of three-dimensional (3D) zincblende and wurtzite crystals, as well as $\mathrm{SiC}$ atomic chain as an ultimate one-dimensional (1D) system; we presented an analysis of optimized atomic structures with corresponding phonon dispersion curves and electronic energy band structures and effective charges. Then we provided an extensive analysis of 2D and quasi-1D (nanoribbon) $\mathrm{SiC}$ in terms of the optimized atomic structures and their stability, electronic, and magnetic structures. We revealed elastic constants, such as in-plane stiffness and Poisson's ratio. Having obtained the results for 1D, 2D, and 3D structures, we presented a comprehensive discussion of dimensionality effects. Then we investigated the effect of vacancy defects (such as $\mathrm{Si}$ and $\mathrm{C}$ vacancy, $\mathrm{Si}+\mathrm{C}$ vacancy and $\mathrm{C}-\mathrm{Si}$ antisite defect) on the electronic and magnetic properties of single layer $\mathrm{SiC}$ and its armchair nanoribbons. Furthermore, we showed that $\mathrm{SiC}$ can be functionalized through adsorption of a foreign atom to the surface of $2 \mathrm{D}$ 
TABLE I. Si-C bond length $d$, lattice constant $a$, bond angle $\alpha$, charge transfer from Si to C $\delta q$, band gap $E_{G}$, and cohesive energy $E_{C}$ values for two different types of $\mathrm{SiC}$ chains.

\begin{tabular}{lcccccc}
\hline \hline Type & $\begin{array}{c}d \\
(\AA)\end{array}$ & $\begin{array}{c}a \\
(\AA)\end{array}$ & $\begin{array}{c}\alpha \\
(\mathrm{deg} .)\end{array}$ & $\begin{array}{c}\delta q \\
(\mathrm{e})\end{array}$ & $\begin{array}{c}E_{G} \\
(\mathrm{eV})\end{array}$ & $\begin{array}{c}E_{C} \\
(\mathrm{eV})\end{array}$ \\
\hline Linear & 1.649 & 3.298 & 180 & 2.28 & 2.00 & 8.962 \\
Wide zigzag & 1.673 & 3.268 & 155.2 & 2.15 & 1.18 & 8.968 \\
\hline \hline
\end{tabular}

$\mathrm{SiC}$ or through substitution of either $\mathrm{C}$ or $\mathrm{Si}$ with a foreign atom. It is revealed that $2 \mathrm{D} \mathrm{SiC}$ and its ribbons provide unusual physical properties, which are absent in 3D SiC crystals. For example, while various allotropic forms of SiC including its honeycomb structures are normally nonmagnetic semiconductors, a Si vacancy gives rise to spin polarization. Significant variation in the band gap of narrow A-SiCNR's with their widths may be crucial in designing optoelectronic nanodevices.

\section{MODEL AND METHODOLOGY}

We have performed first-principles plane-wave calculations within DFT using PAW potentials. ${ }^{17}$ The exchangecorrelation potential has been approximated by generalized gradient approximation (GGA) using PW91 (Ref. 18) functional both for spin-polarized and spin-unpolarized cases. For the sake of comparison, the calculations are also carried out using different potentials and exchange-correlation approximations. All structures have been treated within the supercell geometry using the periodic boundary conditions. A plane-wave basis set with kinetic energy cutoff of $500 \mathrm{eV}$ has been used. A vacuum spacing of $12 \AA$ hinders the interactions between $\mathrm{SiC}$ monolayers in adjacent supercells. In the self-consistent structure optimizations, the Brillouin zone (BZ) is sampled by, respectively, $(5 \times 5 \times 5),(11 \times 11 \times 1)$, and $(11 \times 1 \times 1)$ special $\mathbf{k}$ points for $3 \mathrm{D}$ bulk, 2D honeycomb, and $1 \mathrm{D}$ nanoribbons of SiC. Further relaxation is made with $(11 \times 11 \times 11),(31 \times 31 \times 1)$, and $(25 \times 1 \times 1)$ special $\mathbf{k}$ points in order to find the final structure. All atomic positions and lattice constants are optimized by using the conjugate gradient method, where the total energy and atomic forces are minimized. The convergence for energy is chosen as $10^{-5} \mathrm{eV}$ between two steps and the maximum Hellmann-Feynman forces acting on each atom is less than $0.04 \mathrm{eV} / \AA$ upon ionic relaxation. The pseudopotentials corresponding to four valence electrons of $\mathrm{Si}\left(\mathrm{Si}: 3 s^{2} 3 p^{2}\right)$ and $\mathrm{C}$ $\left(\mathrm{C}: 2 s^{2} 2 p^{2}\right)$ are used. Numerical plane-wave calculations are performed by using VASP. ${ }^{19,20}$ Part of the calculations have also been repeated by using SIESTA (Ref. 21) software. The cohesive energy of any $\mathrm{SiC}$ structure is found as $E_{C}$ $=E_{T}[\mathrm{SiC}]-E_{T}[\mathrm{Si}]-E_{T}[\mathrm{C}]$ in terms of the optimized total energy of any $\mathrm{SiC}$ structure, and the spin-polarized total energies of free $\mathrm{Si}$ and $\mathrm{C}$ atoms, all calculated in the same supercell using the same parameters. Phonon calculations were carried out using PHON program ${ }^{22}$ implementing force constant method. $G W_{0}$ calculations $^{23}$ are again handled by VASP.

\section{1D ATOMIC CHAIN AND 3D BULK CRYSTAL OF SIC}

In this section, we present a brief discussion of $1 \mathrm{D} \mathrm{SiC}$ atomic chain and 3D bulk crystal based on our structure op- timized total energy and phonon spectrum calculations. Studies on $\mathrm{SiC}$ bulk lattice and atomic chains already exist in the literature..$^{2,3,24}$ However, our purpose is to carry out calculations with same parameters as used in $2 \mathrm{D}$ monolayer $\mathrm{SiC}$ honeycomb structure and provide a consistent comparison of dimensionality effects.

\section{A. 1D SiC Chains}

Earlier, the first theoretical study of atomic chains of Groups IV and III-V binary compounds were reported by Senger et al. ${ }^{24}$ They examined $\mathrm{SiC}$ atomic chain as a function of lattice parameter and found that the wide zigzag atomic chain of $\mathrm{SiC}$ with bond angle of $\sim 147^{\circ}$ is energetically more favorable than the linear and narrow angle zigzag chains. Present calculations find that the atomic chains of $\mathrm{SiC}$ are nonmagnetic. Calculated structural parameters, cohesive energies, band gap, and phonon modes of linear and zigzag atomic chains, which are relevant for the present study are given in Table I. The charge transfer from $\mathrm{Si}$ to $\mathrm{C}$ is calculated to be $\delta \mathrm{q}=2.28$ electrons using the Bader analysis. $^{25}$ Phonon modes calculated with force constant method have imaginary frequencies. In Fig. 1 two acoustical and two optical branches of linear SiC chain have imaginary frequencies. Also wide angle zigzag $\mathrm{SiC}$ chain has one optical and one acoustical branch with imaginary frequencies. These results indicate that free standing $\mathrm{SiC}$ chains are not stable. We note that carbon and BN atomic chains are found to be stable and have linear structure. ${ }^{24,26,27}$ Stability of linear chain structure is assured by $\pi$ bonding between adjacent atoms.

\section{B. 3D SiC Crystals}

Our work on bulk SiC includes wurtzite (wz) and zincblende $(\mathrm{zb})$ structures. Atoms in $\mathrm{wz}^{-}$and $\mathrm{zb}-\mathrm{SiC}$ are fourfold coordinated through tetrahedrally directed $s p^{3}$ orbitals. Calculated structural parameters, cohesive energies, energy band structures and phonon modes are given in Fig. 2. Zincblende $\mathrm{SiC}$ structure in $T_{d}$ symmetry has cubic lattice constants, $a_{1}=a_{2}=a_{3}=3.096 \AA$. Si-C bond distance $d$ is $1.896 \AA$. Each $\mathrm{Si}(\mathrm{C})$ is connected to its four nearest neighbor $\mathrm{C}(\mathrm{Si})$; four $\mathrm{Si}-\mathrm{C}$ bonds are equal. Charge transfer from $\mathrm{Si}$ to $\mathrm{C}$ is $\delta q=2.59$ electrons calculated via Bader analysis. ${ }^{25}$ While the GGA band gap is $1.41 \mathrm{eV}$, it increases to $2.40 \mathrm{eV}$ after $G W_{0}$ corrections. As for wz-SiC crystal, the hexagonal lattice constants of the optimized structure in equilibrium are $a_{1}=a_{2}=3.091 \AA, c / a=1.642$. The small deviation of $c / a$ from the ideal value of 1.633 imposes a slight anisotropy on the lengths of tetrahedrally directed Si-C bonds. While the length of three short bonds is $1.893 \AA$, the fourth bond is 


\section{SiC Chains}

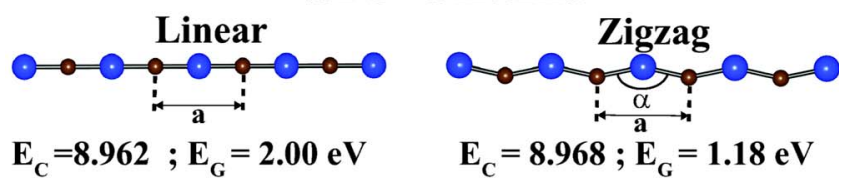

Energy Bands
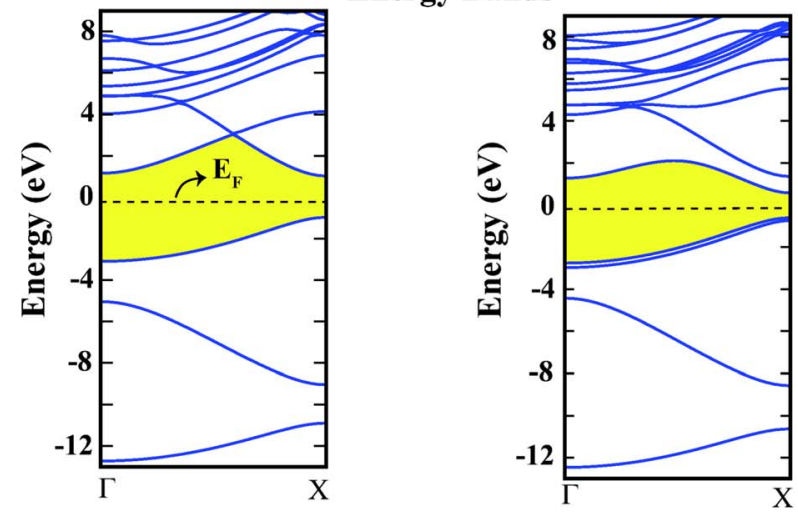

Phonon Modes
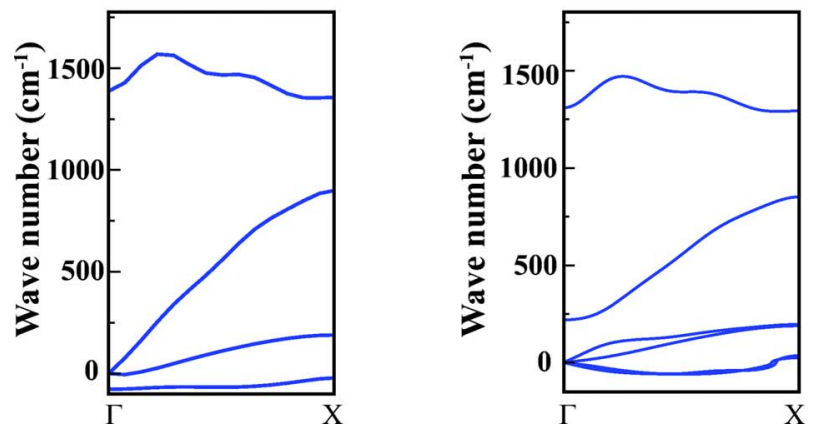

FIG. 1. (Color online) Atomic structures, electronic energy bands, and dispersion of phonon modes of linear and wide angle zigzag $\mathrm{SiC}$ atomic chains. $E_{C}$ and $E_{G}$ are cohesive and band gap energies, respectively. $\mathrm{Si}$ and $\mathrm{C}$ atoms are shown by blue/large and brown/small balls, respectively. Zero of the energy is set to the Fermi energy, $E_{F}$.

slightly longer and has the length of $1.907 \AA$. Charge transfer from $\mathrm{Si}$ to $\mathrm{C}$ is $\delta q=2.63$ electrons. The GGA band gap is $2.36 \mathrm{eV}$, but it increases to $3.32 \mathrm{eV}$ after $G W_{0}$ correction. The calculated structural parameters and energy band gaps are in reasonable agreement with the earlier calculations and experimental measurements. ${ }^{3,28}$ In particular, the band gap values of 2.39 and $3.33 \mathrm{eV}$ for $\mathrm{zb}$ and $\mathrm{wz} \mathrm{SiC}$, respectively are in excellent agreement with the present $G W_{0}$ corrected values.

The frequencies of phonon modes and their dispersions are calculated for $\mathrm{zb}$ and $\mathrm{wz}$ crystals by direct (or force constant) method. ${ }^{22}$ At long wavelengths near the $\Gamma$ point, the electric field due to dipoles is critical for phonon modes. This effect lifts the degeneracy between longitudinal and transverse optical modes. However the splitting (known as LO-TO splitting) cannot be observed with the direct method used in the present study. Therefore, in Fig. 2 the highest and second highest optical branches become degenerate at $\Gamma$ point. Present results are in agreement with earlier phonon calculations. ${ }^{29,30}$

\section{Bulk SiC}

SiC 3D Zincblende

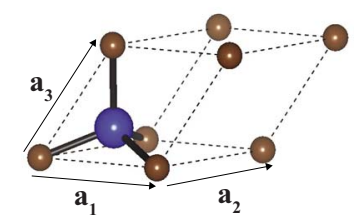

$\mathrm{E}_{\mathrm{C}}=12.939 \mathrm{eV} ; \mathrm{E}_{\mathrm{G}}=1.41 \mathrm{eV}$ $E_{G}\left(+G W_{0}\right)=2.40 \mathrm{eV}$

Energy Bands
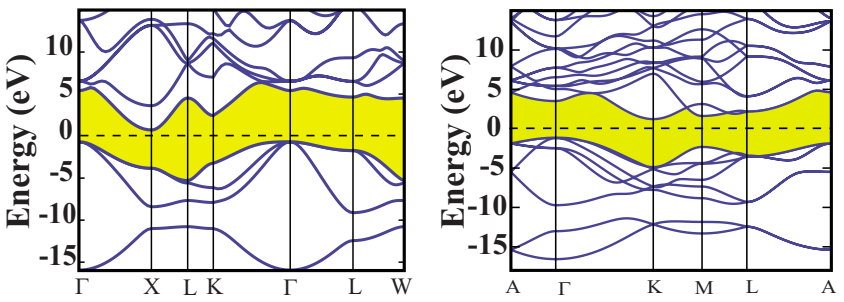

Phonon Modes
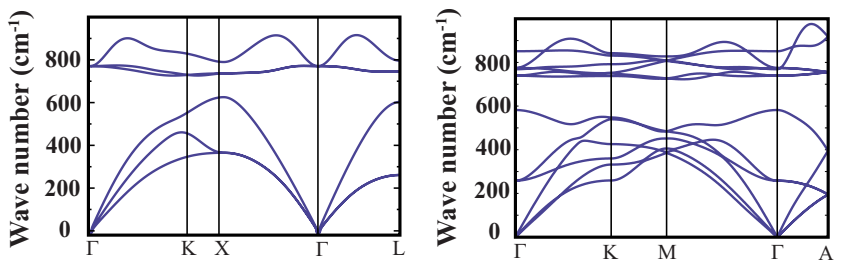

FIG. 2. (Color online) Optimized atomic structure with relevant structural parameters, corresponding energy band structure and frequencies of phonon modes of $3 \mathrm{D}$ bulk $\mathrm{SiC}$ in zincblende and wurtzite structures. Zero of energy of the band structure is set at the Fermi level, and band gap is shaded.

\section{2D SIC HONEYCOMB STRUCTURE}

Two-dimensional monolayer of SiC hexagonal structure of $\mathrm{SiC}$ is optimized using periodically repeating supercell having $12 \AA$ spacing between $\mathrm{SiC}$ planes. The minimum of total energy occurred when $\mathrm{Si}$ and $\mathrm{C}$ atoms are placed in the same plane forming a honeycomb structure. The magnitude of the Bravais lattice vectors of the hexagonal lattice is found to be $a_{1}=a_{2}=3.094 \AA$ (see Fig. 3), and the Si-C bond length to be $d=1.786 \AA$. The planar structure of $2 \mathrm{D} \mathrm{SiC}$ is tested by displacing $\mathrm{Si}$ and $\mathrm{C}$ atoms arbitrarily from their equilibrium positions by $0.5 \AA$ and then reoptimizing the structure. Upon optimization, the displaced atoms returned to their original positions in the same plane implying the stability of planar structure. Further details on the stability of this structure will be provided with phonon calculations at the end of this section. Two-dimensional monolayer $\mathrm{SiC}$ is found to be a semiconductor with a band gap of $2.53 \mathrm{eV}$ within GGA. Furthermore, in Table II, we present lattice constant, bond length, cohesive energy, energy gap values of 2D SiC monolayer calculated with different potentials. Since DFT usually underestimates the band gap of semiconductors, we also corrected the GGA band gap using $G W_{0}$ correction and found it to be $3.90 \mathrm{eV}$. The charge transfer from $\mathrm{Si}$ to $\mathrm{C}$ in $2 \mathrm{D} \mathrm{SiC}$ is calculated to be $\delta \mathrm{q}=2.53$ electrons. The $\mathrm{Si}-\mathrm{C}$ bond length 
(a) Structure

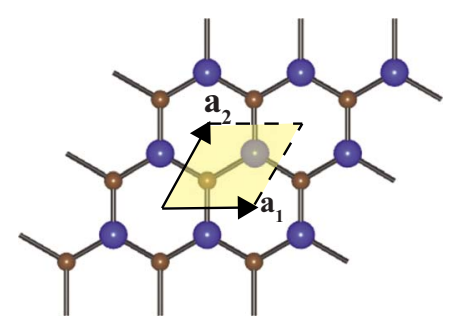

(b) Energy Bands

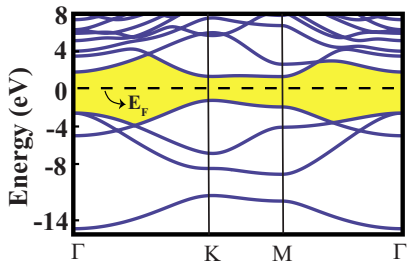

(c) Phonon Dispersions

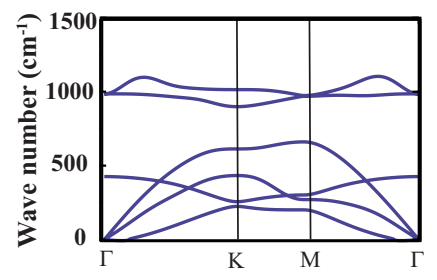

FIG. 3. (Color online) (a) atomic structure, (b) energy band structure, and (c) phonon modes of 2D SiC in honeycomb structure. Large/blue and small/brown balls indicate $\mathrm{Si}$ and $\mathrm{C}$ atoms, respectively. The primitive unit cell is delineated. The zero of energy in the band structure is set to the Fermi level.

and the band gap values in the first row obtained by using GGA+PAW are in agreement with earlier DFT calculations. $^{31}$

In addition to structural and electronic properties, we also investigated the mechanical properties of $2 \mathrm{D} \mathrm{SiC} .^{32-34}$ One can specify the mechanical properties of $\mathrm{SiC}$ honeycomb sheet in terms of uniaxial strain, $\epsilon=\Delta c / c, c$ being the lattice constant; Poisson's ratio $\nu=-\epsilon_{\text {trans }} / \epsilon_{\text {axial }}$ and in-plane stiffness, $C=\frac{1}{A_{0}}\left(\frac{\partial^{2} E_{s}}{\partial \epsilon^{2}}\right)$. Here $A_{0}$ is the equilibrium area of the system and $E_{s}$ is the strain energy calculated by subtracting the total energy of the strained system from the equilibrium total energy. To calculate the elastic constants of monolayer $\mathrm{SiC}$ honeycomb structure we switch to rectangular unit cell and consider a large $(8 \times 4)$ supercell comprising 32 primitive unit cells. In the harmonic region $E_{s}(\epsilon)$ is first calculated on a $2 \mathrm{D}$ grid. The numerical data are then fitted to the expression, $E_{s}=A_{1} \epsilon_{x}^{2}+A_{2} \epsilon_{y}^{2}+A_{3} \epsilon_{x} \epsilon_{y}$; where $\epsilon_{x}$ and $\epsilon_{y}$ are the small strains along $x$ and $y$ directions. In the harmonic region, as a result of the isotropy $A_{1}=A_{2}$. More details concerning the calculation of $C$ and $\nu$ can be found in Refs. 33 and 34. The calculated in-plane stiffness of $\mathrm{SiC}$ honeycomb structure is found to be $166\left(\mathrm{~J} / \mathrm{m}^{2}\right)$. This is almost half of the in-plane stiffness of graphene (namely $335 \mathrm{~J} / \mathrm{m}^{2}$ ), but more than twice the in-plane stiffness of silicene $\left(62 \mathrm{~J} / \mathrm{m}^{2}\right)$. Also the Poisson's ratio of $\mathrm{SiC}$ is calculated to be 0.29 . Consequently, 2D SiC monolayer is a stiff material, but less stiff than graphene and BN having similar honeycomb structures.

Similar to $1 \mathrm{D}$ and $3 \mathrm{D} \mathrm{SiC}$, frequencies of phonon modes of and the dispersions $\Omega(\mathbf{k})$ of $2 \mathrm{D}$ monolayer of $\mathrm{SiC}$ in planar geometry are calculated using direct method. ${ }^{22}$ Forces were found by displacing a single atom in a $7 \times 7 \times 1$ supercell. We use a small displacement in order to stay in the harmonic region. We increased default grids used by VASP until calculations converge. The lowest acoustical mode, which is called as the out of plane, ZA mode is vulnerable to instability. In rough meshes this mode gets imaginary frequencies near the $\Gamma$ point, but it can be overcome by refining the mesh along the $z$ axis (perpendicular to plane) as much as possible. This way, force in that direction is calculated more rigorously. Since the frequencies of all modes are positive in $\mathrm{BZ}$, it is concluded that planar, 2D SiC monolayer in honeycomb structure is stable.

\section{Dimensionality effects}

In Table III, we compare the variation in the effective charge on $\mathrm{Si}$ and $\mathrm{C}$ atoms, namely $Z_{\mathrm{Si}}^{*}$ and $Z_{\mathrm{C}}^{*}$, respectively; charge transfer from $\mathrm{Si}$ to $\mathrm{C}, \delta q=4-Z_{\mathrm{Si}}^{*}$; Si-C bond length $d$; lattice constant; energy band gap, $G W_{0}$ corrected band gap and cohesive energy per $\mathrm{Si}-\mathrm{C}$, calculated for $\mathrm{SiC}$ in different dimensionalities. It should be noted that the length of $\mathrm{Si}-\mathrm{C}$ bonds of $2 \mathrm{D} \mathrm{SiC} \mathrm{honeycomb} \mathrm{structure} \mathrm{is} \mathrm{smaller} \mathrm{than} \mathrm{that} \mathrm{in}$ the 3D bulk (wz, zb) crystals, but larger than that in zigzag atomic chains. Here we see that the dimensionality effect is reflected to the strength of the bonding through $s p^{n}$ hybridization, where $n$ coincides with the dimensionality. While $s p^{2}$ hybrid orbitals of 2D planar honeycomb structure form stronger bonds than tetrahedrally coordinated $s p^{3}$ orbitals of $3 \mathrm{D}$ bulk, they are relatively weaker than $s p$ hybrid orbitals of 1D chain. Accordingly, $d$ is shortest in 1D chain, and longest in $3 \mathrm{D} \mathrm{zb}$ structures, and is intermediate in $2 \mathrm{D}$ monolayer. The cohesive energy $E_{C}$ increases with dimensionality, since the number of nearest-neighbors increases. Effective charge or charge transfer between cation and anion also varies with

TABLE II. Si-C bond length $d$, lattice constant $a$, band gap $E_{G}$, band gap corrected by $G W_{0} E_{G W_{0}}$, cohesive energy $E_{C}$ values for $2 \mathrm{D}$ monolayer of $\mathrm{SiC}$ in honeycomb structure calculated with different potentials.

\begin{tabular}{lccccc}
\hline \hline Potential & $\begin{array}{c}d \\
\AA\end{array}$ & $\begin{array}{c}a \\
(\AA)\end{array}$ & $\begin{array}{c}E_{G} \\
(\mathrm{eV})\end{array}$ & $\begin{array}{c}E_{G}\left(+G W_{0}\right) \\
(\mathrm{eV})\end{array}$ & $\begin{array}{c}E_{c} \\
(\mathrm{eV})\end{array}$ \\
\hline PAW+GGA & 1.786 & 3.094 & 2.530 & 3.90 & 11.944 \\
PAW+LDA & 1.770 & 3.070 & 2.510 & & 13.542 \\
US+GGA & 1.776 & 3.079 & 2.542 & & 11.973 \\
US+LDA & 1.759 & 3.048 & 2.532 & & 13.471 \\
\hline \hline
\end{tabular}


TABLE III. Bonding types, Si-C distances $d$, lattice constants $a$, charge transfers $\delta q$, effective charges on $\mathrm{Si}$ and $\mathrm{C}$, respectively, $Z_{\mathrm{Si}}^{*}$ and $Z_{\mathrm{C}}^{*}$, band gaps $E_{G}$ with $G W_{0}$ corrections, and cohesive energies $E_{c}$ for comparison of SiC polymorphs.

\begin{tabular}{|c|c|c|c|c|c|c|c|c|c|}
\hline Structure & Bonding & $\begin{array}{c}d \\
(\AA)\end{array}$ & $\begin{array}{c}a \\
(\AA)\end{array}$ & $\begin{array}{l}\delta q \\
(\mathrm{e})\end{array}$ & $\begin{array}{l}Z_{\mathrm{Si}}^{*} \\
(\mathrm{e})\end{array}$ & $\begin{array}{l}Z_{\mathrm{C}}^{*} \\
(\mathrm{e})\end{array}$ & $\begin{array}{c}E_{G} \\
(\mathrm{eV})\end{array}$ & $\begin{array}{c}E_{G}\left(+G W_{0}\right) \\
\quad(\mathrm{eV})\end{array}$ & $\begin{array}{c}E_{C} \\
(\mathrm{eV})\end{array}$ \\
\hline Linear chain & $s p$ & 1.649 & 3.298 & 2.28 & 1.72 & 6.28 & 2.00 & & 8.923 \\
\hline Wide zigzag chain & $s p$ & 1.673 & 3.268 & 2.15 & 1.85 & 6.15 & 1.18 & & 8.963 \\
\hline 2D honeycomb & $s p^{2}$ & 1.786 & 3.094 & 2.53 & 1.47 & 6.53 & 2.53 & 3.90 & 11.940 \\
\hline Zincblende & $s p^{3}$ & 1.896 & 3.096 & 2.59 & 1.41 & 6.59 & 1.41 & 2.40 & 12.939 \\
\hline Wurtzite & $s p^{3}$ & $1.893(3), 1.907(1)$ & 3.091 & 2.63 & 1.37 & 6.63 & 2.36 & 3.32 & 12.933 \\
\hline
\end{tabular}

dimensionality. For example, the charge transfer calculated with Bader analysis increases with increasing dimensionality. While the energy band gap $E_{G}$ does not show a regular trend with dimensionality, the band gap of 2D monolayer is wider than those of 3D crystals.

\section{BARE AND HYDROGEN PASSIVATED SIC NANORIBBONS}

In this section, we consider bare and hydrogen passivated armchair SiC nanoribbons. These nanoribbons are specified according to their widths specified in terms of $N$ number of $\mathrm{Si}-\mathrm{C}$ basis in their unit cells. Hence, $\mathrm{A}-\operatorname{SiCNR}(N)$ indicates armchair $\mathrm{SiC}$ nanoribbons having $N$ Si-C pairs in their unit cell. We have analyzed A-SiCNR (both bare and H-passivated) from $N=5$ to 21. A-SiCNR's with odd numbered $N$ have reflection symmetry with respect to their axis. Bare armchair $\mathrm{SiC}$ nanoribbons are ferromagnetic in ideal honeycomb form. However, upon structural relaxation, reconstruction occurs at the edges resulting in a considerable gain of energy and the structure becomes nonmagnetic.

Band gaps of all A-SiCNR(N) increase by $\sim 0.7 \mathrm{eV}$ upon $\mathrm{H}$ saturation of dangling bonds. The $\mathrm{Si}-\mathrm{H}$ and $\mathrm{C}-\mathrm{H}$ bonds formed after hydrogenation have lengths of 1.49 and $1.09 \AA$, respectively. The effective charges on $\mathrm{Si}$ and $\mathrm{C}$ edge atoms changes after $\mathrm{H}$ passivation. While $\mathrm{Si}-\mathrm{C}$ bonds at the edges are shorter than the $\mathrm{Si}-\mathrm{C}$ bonds in $2 \mathrm{D} \mathrm{SiC}$ monolayer by $0.09 \AA$, upon $\mathrm{H}$ saturation these bonds get slightly longer, but are still shorter than the regular $\mathrm{Si}-\mathrm{C}$ bonds by $0.05 \AA$. Hence, reconstruction of atomic structure at the edge exists in each case, but is more pronounced in the bare nanoribbons.

Here we consider A-SiCNR(9) as a prototype and examine its band structure. The bare A-SiCNR(9) is an indirect band gap semiconductor. Two bands at the conduction band edge are composed of edge states, which are split due to edge-edge interactions. These bands are removed upon $\mathrm{H}$ saturation of dangling bonds of atoms at the edges. This results in a widening of the band gap. As for the other edge state band, it is located in the valance band. This band is also removed upon $\mathrm{H}$-saturation, but the valence band edge is not affected. The band gap of H-saturated A-SiCNR(9) is direct. Energy bands and band decomposed charge densities are shown in Fig. 4.

The variation of the energy band gap, $E_{G}$, of bare and $\mathrm{H}$-passivated $\mathrm{SiC}$ armchair nanoribbons with the width of the ribbon $N$ are calculated for $5 \leq \mathrm{N} \leq 21$ as presented in Fig. 5 . For bare A-SiCNR's, the band gap is relatively smaller due to edge states as shown in Fig. 4; namely, $E_{G} \sim 1.29 \mathrm{eV}$ for $N=5$, but increases to $\sim 2.4 \mathrm{eV}$ for $N=21$. Upon $\mathrm{H}$ passivation of the dangling bonds at the edges, the edge states disappear and the band gap increases and gets direct. For $N$ $=5$ the direct band gap is around $2.5 \mathrm{eV}$, but increases with $\mathrm{N}$ and eventually becomes $2.38 \mathrm{eV}$ for $N=21$. Hypothetically, the band gap is expected to reach the value of $2 \mathrm{D}$ planar $\mathrm{SiC}$ $\left(E_{G}=2.53 \mathrm{eV}\right.$ calculated within GGA). The main difference with graphene is that the band gap of bare armchair graphene nanoribbon decreases with increasing $N$ and eventually vanish as $N \rightarrow \infty$. Interestingly, $\mathrm{H}$-saturated armchair SiCNRs with, $N-1, N$, and $N+1$, exhibit a family behavior similar to one revealed in armchair graphene nanoribbons. ${ }^{35}$ For $N$ $>15$ the variation of $E_{G}$ of H-saturated A-SiCNR is not significant. However, it should be noted that the band gap variation of both bare and $\mathrm{H}$-saturated armchair SiC nanoribbons cannot be reconciled with the quantum confinement effects, since $E_{G}$ increases with increasing $N$. This is due to other effects which overcome the quantum confinement effect. The variation in band gaps of bare and hydrogen passivated arm- (a) Bare A-SiCNR (9)

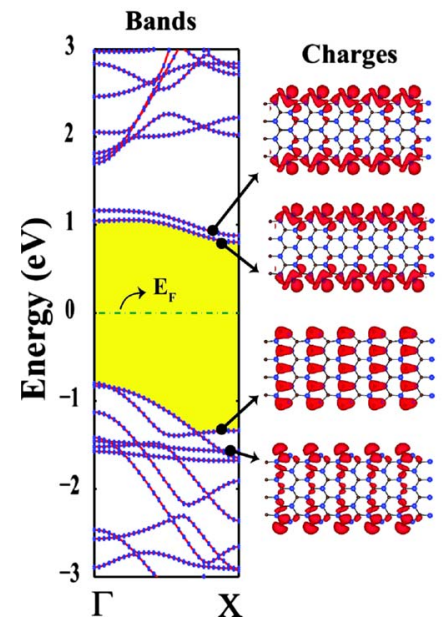

(b) H-passivated A-SiCNR (9)

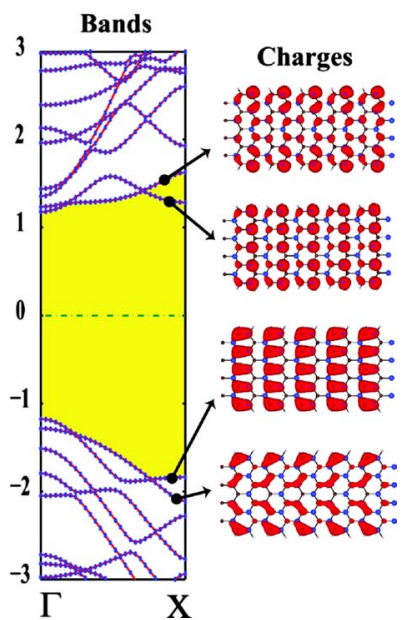

FIG. 4. (Color online) Energy band structure of the bare (a) and hydrogen saturated (b) armchair $\mathrm{SiC}$ nanoribbons, A-SiCNR $(N)$ having $9 \mathrm{Si}-\mathrm{C}$ pairs $(N=9)$ in the unit cell and isosurfaces of charge densities of selected states at the $\Gamma$-point of BZ. Zero of energy is set at the Fermi level. Energy band gap is shaded. 
(a)

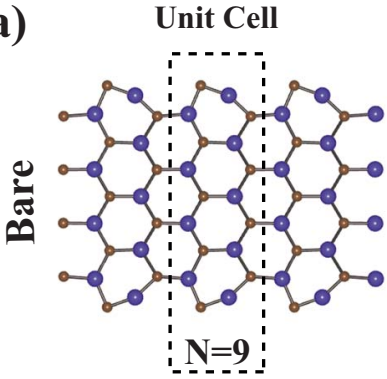

(c)





(d)

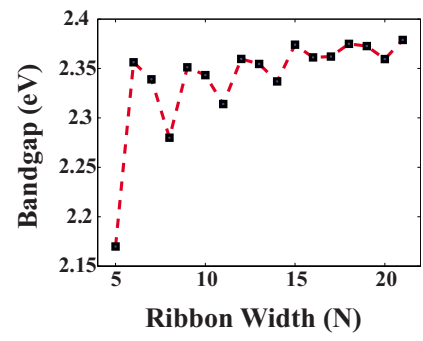

FIG. 5. (Color online) Optimized atomic structures for (a) Bare (b) H-passivated $\mathrm{SiC}$ nanoribbons. Large/blue and small/brown balls represent $\mathrm{Si}$ and $\mathrm{C}$ atoms, respectively. The variations of band gaps of (c) Bare and (d) H-passivated $\mathrm{SiC}$ nanoribbons with the ribbon width $\mathrm{N}$ for $5<N<21$.

chair $\mathrm{SiC}$ ribbons are presented in Fig. 5(c) and 5(d). In Ref. 31 , the variation of the band gap with $\mathrm{N}$ and resulting family behavior is in agreement with present results. Whereas the same variation is not presented in Ref. 31. The zigzag SiCNRs exhibit interesting magnetic properties and were investigated extensively by earlier works. ${ }^{31,36,37}$ For this reason, the zigzag SiCNRs are examined in the present study.

\section{VACANCY DEFECTS AND ANTISITE}

It has been shown that the vacancy defects have remarkable effects on 2D graphene honeycomb structure and its nanoribbons. ${ }^{38-43}$ Nonmagnetic graphene sheets or nanoribbons can attain spin polarized states due to vacancy defects. We expect that similar effects of vacancy defects can occur on the electronic and magnetic properties of $\mathrm{SiC}$ honeycomb structure.

\section{A. 2D Honeycomb SiC}

The effects of $\mathrm{Si}$ and $\mathrm{C}$ vacancies, $\mathrm{Si}+\mathrm{C}$-divacancy and $\mathrm{C}$-Si-antisite are treated in periodically repeating supercells. The size of supercell is optimized to allow negligible defectdefect interaction between adjacent cells. Here the width of the flat bands derived from the states of periodically repeating vacancies is taken as the measure of the strength of vacancy-vacancy coupling. A $(7 \times 7)$ supercell is found to be suitable, since it leads to rather flat defect bands. At the same time it is not large and allows us to carry out numerical calculations within feasible computational time. The flat bands associated with vacancies can be considered as localized defect state (if it is in the band gap) or resonance state
Vacancies and Antisite in 7x7 2D SiC



(c) $\mathrm{Si}+\mathrm{C}$ Vacancy

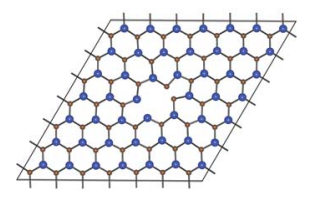

$\mu=0$ (b) Si Vacancy



(d) Antisite

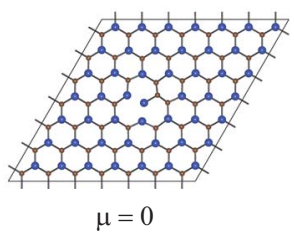

FIG. 6. (Color online) Optimized atomic structure and magnetic moment of vacancy defects calculated in a $(7 \times 7)$ supercell of $2 \mathrm{D}$ $\mathrm{SiC}$ structure. (a) C vacancy; (b) Si vacancy; (c) C+Si divacancy; (d) $\mathrm{C}$-Si antisite. In (b) the difference of spin-up and spin-down charges are shown. Large/blue and small/brown balls indicate $\mathrm{Si}$ and $\mathrm{C}$ atoms respectively.

(if it is in the band continua). Here we consider localized defect states. Our results are presented in Fig. 6 for single C, $\mathrm{Si}$ vacancies, $\mathrm{Si}+\mathrm{C}$ divacancy, $\mathrm{C}+\mathrm{Si}$ antisite defects.

A vacancy is generated first by removing a single atom, C or $\mathrm{Si}$ atom from each supercell of the monolayer of $\mathrm{SiC}$ as shown in Figs. 6(a) and 6(b). Subsequently, the atomic structure is optimized. Single C vacancy in 2D monolayer of $\mathrm{SiC}$ is nonmagnetic; $\mathrm{Si}$ atoms around vacancy with coordination number 2 are displaced in the transversal direction and do not induce any magnetic moment.

As for $\mathrm{Si}$ vacancy, three $\mathrm{C}$ atoms around vacancy remained planar. Similar to the vacancies in graphene and BN, Si-vacancy induces a local magnetization in the system. Isovalue surfaces of the difference between spin-up and spindown charge densities i.e., $\Delta \rho^{\uparrow \downarrow}$ clearly shows a spin polarization around the vacancy and a net magnetic moment constructed there from. The calculated total magnetic moment is $4 \mu_{B}$ per supercell. The Si vacancy in a repeating $(7 \times 7)$ give rise to defect states in the band gap. As for $\mathrm{Si}$ $+\mathrm{C}$ divacancy in Fig. 6(c), it is again nonmagnetic since the spins are paired; two $\mathrm{C}$ atoms around the vacancy choose to make a bond with each other. The band gap is also modified. Finally, we consider the antisite defect. The resulting relaxed structure is given in Fig. 6(d). Lattice is distorted as C-C bond is shorter than $\mathrm{Si}$-Si bond in the antisite case. It is noted that the calculated magnetic moments for single $\mathrm{Si}$ and $\mathrm{C}$ vacancy do not agree with Lieb's theorem, ${ }^{44}$ which normally predicts $1 \mu_{B}$ net magnetic moment both for $\mathrm{Si}$ and $\mathrm{C}$ vacancies in Fig. 6. We attribute the discrepancy between the results of first principles calculations and Lieb's theorem to the structural relaxation occurred after the generation of vacancy and significant charge transfer from $\mathrm{Si}$ to $\mathrm{C}$. The localized electronic states associated with the vacancy defects and antisite are deduced from the band structure calculations as presented in Table IV, where the energies are given from the top of the valance band. 
TABLE IV. Magnetic moments and positions of defect-induced state energies relative to the top of the valance band. Spin-up $(\uparrow)$ and spin-down $(\downarrow)$ states are indicated. E (F) indicate whether the defect state is empty (full-occupied).

\begin{tabular}{|c|c|c|c|c|c|}
\hline \multirow{3}{*}{$\frac{\mu}{E_{1}(\mathrm{eV})}$} & \multirow{3}{*}{$\begin{array}{c}\text { Si }+ \text { C vacancy } \\
0 \\
0.49(\mathrm{~F})\end{array}$} & \multirow{3}{*}{$\begin{array}{c}\text { C-vacancy } \\
0 \\
0.15(\mathrm{~F})\end{array}$} & \multicolumn{2}{|c|}{ Si-vacancy } & \multirow{3}{*}{$\begin{array}{c}\text { Antisite } \\
0 \\
0.59(\mathrm{~F})\end{array}$} \\
\hline & & & \multicolumn{2}{|c|}{$4 \mu_{B}$} & \\
\hline & & & 0.09 & $(\mathrm{~F} \uparrow)$ & \\
\hline$E_{2}$ & $1.40(\mathrm{E})$ & $1.82(\mathrm{E})$ & 0.11 & $(\mathrm{~F} \uparrow)$ & $2.12(\mathrm{E})$ \\
\hline$E_{3}$ & $2.45(\mathrm{E})$ & $1.95(\mathrm{E})$ & 0.31 & $(\mathrm{~F} \downarrow)$ & \\
\hline$E_{4}$ & & & 0.33 & $(\mathrm{~F} \downarrow)$ & \\
\hline$E_{5}$ & & & 0.38 & $(\mathrm{E} \downarrow)$ & \\
\hline$E_{6}$ & & & 1.47 & $(\mathrm{E} \downarrow)$ & \\
\hline$E_{7}$ & & & 1.49 & $(\mathrm{E} \downarrow)$ & \\
\hline
\end{tabular}

\section{B. Vacancy defects in SiC Nanoribbons}

The effects of the vacancy defects on the electronic and magnetic properties are treated for of $\mathrm{H}$-passivated A-SiCNR $(9)$ using a $(4 \times 1)$ repeating supercell. Our main motivation was to investigate what differences would occur in a ribbon. Optimized structures, calculated total magnetic moments are presented in Fig. 7. Overall effects of vacancy, divacancy and antisite effects are similar to those in 2D SiC monolayer structure except in the antisite case the exchanged $\mathrm{Si}$ atom moves out of the plane about $0.9 \AA$. One localized state below the conduction band edge and one other state above the valence band edge occur due to antisite as donor and acceptor states, respectively.

\section{FUNCTIONALIZATION OF SIC HONEYCOMB STRUCTURE BY ADATOMS}

Specific adatoms can bind to 2D SiC monolayer with significant binding energies. Adatom adsorption or decoration,

Vacancy and Antisite in A-SiC NR (9) (a) C Vacancy

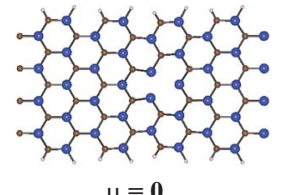

(c) $\mathrm{Si}+\mathrm{C}$ Divacancy

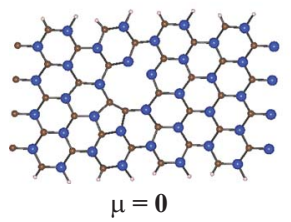

(b) Si Vacancy

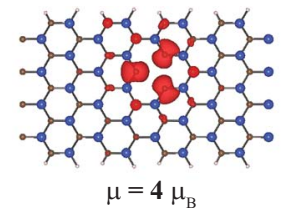

(d) Antisite

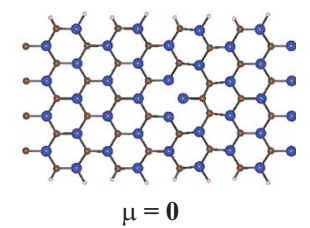

FIG. 7. (Color online) Magnetic moment of vacancy defects calculated in a $(4 \times 1)$ supercell of quasi $1 \mathrm{D} \mathrm{SiC}$ armchair nanoribbon with $N=9$, i.e., A-SiCNR(9). Dangling bonds at both edges are saturated by hydrogen atoms. (a) C vacancy; (b) Si vacancy; (c) $\mathrm{Si}+\mathrm{C}$ divacancy; (d) C-Si antisite. In (b) the difference of spin-up and spin-down charges are shown while others are nonmagnetic. Large/blue and small/brown balls represent $\mathrm{Si}$ and $\mathrm{C}$ atoms respectively. as well as substitution for $\mathrm{Si}$ or $\mathrm{C}$ atoms in the honeycomb structure by foreign atoms can modify the properties of $2 \mathrm{D}$ $\mathrm{SiC}$ monolayer and its nanoribbons. This way, SiC honeycomb structures can be functionalized. Adatom adsorption and substitution are considered within the periodically repeating $(7 \times 7)$ supercell geometry to minimize the interaction between them. The calculations performed in a larger supercell $(10 \times 10)$ have also showed similar results which is an indication that the coupling between adjacent defects is negligible.

\section{A. Adatom adsorption}

As for adatom adsorption, we considered Al, Co, Fe, N, P, Ti by placing each of them on four different positions in the $(7 \times 7)$ monolayer of $\mathrm{SiC}$ and then by fully relaxing the whole system. The initial positions of the adsorption are on top of silicon atom (TS), on top of carbon atom (TC), at the center of hexagon (HS), above the middle of the $\mathrm{Si}-\mathrm{C}$ bond (BS). The distance between adatoms is $12 \AA$. Spin polarized calculations are carried out to determine the binding structure and binding energy. Whether the adatoms are bound to the surface are examined by calculating the binding energies of these six different individual atoms in terms of the calculated total energies as $E_{B}=E_{T}[\mathrm{SiC}+$ adatom $]-E_{T}[\mathrm{SiC}($ bare $)]$ $-E_{T}$ [adatom $]$. We found that all of these adatoms are bound with a significant energy, which is larger than $1 \mathrm{eV}$. Flat bands indicate that states induced by the adatoms are rather localized and hence adatom-adatom interactions are negligible. Therefore, adatoms treated here in supercell geometry can represent single (isolated) adatom. Optimized adsorption sites, total magnetic moments, heights from the SiC plane are given in Table V.

\section{B. Substitution of $\mathrm{Si}$ and $\mathrm{C}$ by foreign atoms}

Here we examined the substitution of single $\mathrm{Si}$ or $\mathrm{C}$ atoms in $2 \mathrm{D} \mathrm{SiC}$ honeycomb structure by various foreign atoms. Namely, B and $\mathrm{N}$ substituting $\mathrm{C}$ atom; Al, As, Ga, P substituting $\mathrm{Si}$ atom. Similar to adatom calculations, the substitution process is treated within periodically repeating $(7 \times 7)$ supercell. Because of periodic boundary condition the localized states appear as flat bands. We found that B, N, As, and 
TABLE V. Binding energy $E_{B}$, total magnetic moments $\mu$, optimized heights of adatoms from the 2D monolayer of $\mathrm{SiC} h$, and the energies of the localized states occurring in the band gap are given for each type of adatom. Empty (E), full (F), spin-up and spin-down states are indicated. Energies of adatom induced localized states are given relative to the top of the valence band.

\begin{tabular}{|c|c|c|c|c|c|c|c|c|}
\hline & \multicolumn{2}{|c|}{$\mathrm{Co}$} & \multicolumn{2}{|c|}{$\mathrm{Fe}$} & \multicolumn{2}{|c|}{$\mathrm{P}$} & \multicolumn{2}{|c|}{$\mathrm{Ti}$} \\
\hline Position & \multicolumn{2}{|c|}{ HS } & \multicolumn{2}{|c|}{ HS } & \multicolumn{2}{|c|}{ BS } & \multicolumn{2}{|c|}{$\mathrm{TS}$} \\
\hline$E_{B}(\mathrm{eV})$ & \multicolumn{2}{|c|}{2.099} & \multicolumn{2}{|c|}{1.928} & \multicolumn{2}{|c|}{1.758} & \multicolumn{2}{|c|}{2.605} \\
\hline$\mu\left(\mu_{B}\right)$ & \multicolumn{2}{|c|}{1} & \multicolumn{2}{|c|}{2} & \multicolumn{2}{|c|}{1} & \multicolumn{2}{|c|}{2} \\
\hline$h(\AA)$ & \multicolumn{2}{|c|}{1.392} & \multicolumn{2}{|c|}{1.465} & \multicolumn{2}{|c|}{1.743} & \multicolumn{2}{|c|}{1.355} \\
\hline$E_{1}(\mathrm{eV})$ & 0.13 & $(\mathrm{~F} \uparrow)$ & 0.30 & $(\mathrm{~F} \uparrow)$ & 0.17 & $(\mathrm{~F} \uparrow)$ & 0.43 & $(\mathrm{~F} \uparrow)$ \\
\hline$E_{2}$ & 0.23 & $(\mathrm{~F} \uparrow)$ & 0.31 & $(\mathrm{~F} \uparrow)$ & 0.16 & $(\mathrm{~F} \uparrow)$ & 0.60 & $(\mathrm{~F} \downarrow)$ \\
\hline$E_{3}$ & 0.33 & $(\mathrm{~F} \downarrow)$ & 0.82 & $(\mathrm{~F} \downarrow)$ & 0.20 & $(\mathrm{~F} \downarrow)$ & 1.62 & $(\mathrm{~F} \uparrow)$ \\
\hline$E_{4}$ & 0.41 & $(\mathrm{~F} \downarrow)$ & 0.82 & $(\mathrm{~F} \downarrow)$ & 0.20 & $(\mathrm{~F} \uparrow)$ & 1.62 & $(\mathrm{~F} \uparrow)$ \\
\hline$E_{5}$ & 0.67 & $(\mathrm{~F} \uparrow)$ & 1.17 & $(\mathrm{~F} \downarrow)$ & 0.31 & $(\mathrm{~F} \downarrow)$ & 1.99 & $(\mathrm{E} \uparrow)$ \\
\hline$E_{6}$ & 0.80 & $(\mathrm{~F} \downarrow)$ & 1.93 & $(\mathrm{E} \uparrow)$ & 0.33 & $(\mathrm{E} \downarrow)$ & 2.28 & $(\mathrm{E} \uparrow)$ \\
\hline$E_{7}$ & 1.26 & $(\mathrm{E} \downarrow)$ & 2.20 & $(\mathrm{E} \downarrow)$ & & & 2.40 & $(\mathrm{E} \downarrow)$ \\
\hline
\end{tabular}

$\mathrm{P}$ atoms have a net magnetic moment $\mu=1 \mu_{B}$. Ga and $\mathrm{Al}$ do not create any spin polarization. In Table VI, the substitutional impurity states in the band gap of $2 \mathrm{D} \mathrm{SiC}$ and resulting net magnetic moments are given. The substitutional foreign atoms, namely B, N, As, and P have either 3 or 5 valence electrons and hence destroy the spin pairing in the perfect honeycomb structure. The substitution of $\mathrm{C}$ and $\mathrm{Si}$ by these atoms gives rise to a net magnetic moment of $1 \mu_{B}$. As a result, $2 \mathrm{D} \mathrm{SiC}$ can be magnetized without doping by transition metal elements.

\section{DISCUSSION AND CONCLUSIONS}

We present a study on $1 \mathrm{D} \mathrm{SiC}$ chains, 2D monolayer of $\mathrm{SiC}$ in honeycomb structure and its armchair nanoribbons, 3D bulk $\mathrm{SiC}$. We carried out stability analysis of those materials. Two-dimensional monolayer of $\mathrm{SiC}$ is an ionic compound with charge transfer from silicon atoms to carbon atoms and has a hexagonal lattice forming a planar honeycomb structure. The calculation of phonon modes results in all positive frequencies in $\mathrm{BZ}$ and indicates the stability of the planar honeycomb structure. This situation is in compliance with the previous works, ${ }^{16,32}$ where the honeycomb structures of binary compounds of group IV elements or III-V compounds are stable in planar geometry if they have an element from the first row, such as $\mathrm{B}, \mathrm{C}, \mathrm{N}$. The $2 \mathrm{D} \mathrm{SiC}$ is a nonmagnetic wide band gap semiconductor. However, it acquires net magnetic moment when a single Si-vacancy defect is created or $\mathrm{Si}$ and $\mathrm{C}$ atoms of the honeycomb structure are substituted by As, P, B, and N. Single C-vacancy, $\mathrm{Si}+\mathrm{C}$ divacancy and $\mathrm{Si}-\mathrm{C}$ antisite defects does not give rise to any magnetic moment in the system. It is shown that $2 \mathrm{D} \mathrm{SiC} \mathrm{can}$ be functionalized through $\mathrm{Si}$-vacancy and adatom adsorption or substitution of $\mathrm{Si} \mathrm{C}$ by foreign atoms.

Armchair SiC nanoribbons are found to be nonmagnetic semiconductors. We revealed the variation of band gap with the width of both bare and H-passivated nanoribbons. The variation of the band gap exhibits also family behavior. However, the band gaps of armchair nanoribbons are smaller than the band gap of $2 \mathrm{D} \mathrm{SiC}$ for small $\mathrm{N}$. Therefore the confinement effect seen in narrow graphene armchair nanoribbons does not occur here.

In conclusion, our state-of-the-art first-principles calculations demonstrate that $2 \mathrm{D} \mathrm{SiC}$ monolayer is stable in honeycomb structure, and hence it has a strong chance to be synthesized in future. Above results indicate that bare and $\mathrm{H}$-passivated $\mathrm{SiC}$ sheets and armchair nanoribbons can present interesting properties which can be utilized in nanotechnology. Creating defects through vacancies, doping through adatoms and substitutional impurities can functionalize $\mathrm{SiC}$ honeycomb structure and hence add new magnetic and electronic properties.

TABLE VI. Energies of defects states occurring in the band gap are given for each type of substituted atom. Empty (E), full (F), spin-up and spin-down states are indicated. Energies are measured from the top of valence band.

\begin{tabular}{|c|c|c|c|c|c|c|c|c|}
\hline \multirow{3}{*}{$\frac{\mu\left(\mu_{B}\right)}{E_{1}(\mathrm{eV})}$} & \multicolumn{2}{|c|}{ B } & \multicolumn{2}{|c|}{$\mathrm{N}$} & \multicolumn{2}{|c|}{ As } & \multicolumn{2}{|c|}{$\mathrm{P}$} \\
\hline & \multicolumn{2}{|c|}{1} & \multicolumn{2}{|c|}{1} & \multicolumn{2}{|c|}{1} & \multicolumn{2}{|c|}{1} \\
\hline & 0.23 & $(\mathrm{~F}, \uparrow)$ & 1.47 & $(\mathrm{~F}, \uparrow)$ & 0.75 & $(\mathrm{~F}, \uparrow)$ & 1.18 & $(\mathrm{~F}, \uparrow)$ \\
\hline$E_{2}$ & 0.69 & $(\mathrm{E}, \downarrow)$ & 2.37 & $(\mathrm{E}, \downarrow)$ & 1.18 & $(\mathrm{E}, \downarrow)$ & 1.77 & $(\mathrm{E}, \downarrow)$ \\
\hline
\end{tabular}




\section{ACKNOWLEDGMENTS}

We would like to thank Dario Alfè for his valuable discussions. Part of the computations has been carried out with the service provided by UYBHM at Istanbul Technical University through a Grant No. 2-024-2007. This work is partially supported by TUBA, Academy of Science of Turkey. *ciraci@fen.bilkent.edu.tr

${ }^{1}$ Silicon Carbide: Recent Major Advances, edited by W. J. Choyke, H. Matsunami, and G. Pensl (Springer-Verlag, Berlin, 2004).

${ }^{2}$ A. R. Lubinsky, D. E. Ellis, and G. S. Painter, Phys. Rev. B 11, 1537 (1975).

${ }^{3}$ W. Y. Ching, Yong-Nian Xu, Paul Rulis, Lizhi Ouyang, Mater. Sci. Eng. 422, 147 (2004).

${ }^{4}$ We. Zhou, L. Yan, Y. Wang, and Y. Zhang, Appl. Phys. Lett. 89, 013105 (2006).

${ }^{5}$ A. Miranda, J. L. Cueva, A. E. Ramos, and M. Cruz-Irisson, Microelectron. J. 40, 796 (2009).

${ }^{6}$ J. Q. Hu, Y. Bando, J. H. Zhan, and D. Golberg, Appl. Phys. Lett. 85, 2932 (2004).

${ }^{7}$ K. J. Kim, S. Lee, J. H. Lee, M.-H. Roh, K.-Y. Lim, and Y.-W. Kim, J. Am. Ceram. Soc. 92, 424 (2009).

${ }^{8}$ G. N. Yushin, Z. G. Cambaz, Y. Gogotsi, K. L. Vyshnyakova, and L. N. Pereselentseva, J. Am. Ceram. Soc. 91, 83 (2008).

${ }^{9}$ W. Shan, A. J. Fischer, J. J. Song, G. E. Bulman, H. S. Kong, M. T. Leonard, W. G. Perry, M. D. Bremser, and R. F. Davis, Appl. Phys. Lett. 69, 740 (1996).

${ }^{10}$ S. F. Chichibu, M. Sugiyama, T. Kuroda, A. Tackeuchi, T. Kitamura, H. Nakanishi, T. Sota, S. P. DenBaars, S. Nakamura, S. P. DenBaars, and S. Nakamura, Appl. Phys. Lett. 79, 3600 (2001).

${ }^{11}$ N. Camara, G. Rius, J.-R. Huntzinger, A. Tiberj, N. Mestres, P. Godignon, and J. Camassel, Appl. Phys. Lett. 93, 123503 (2008).

${ }^{12}$ J. Hou and B. Songa, J. Chem. Phys. 128, 154304 (2008).

${ }^{13}$ K. S. Novoselov, A. K. Geim, S. V. Morozov, D. Jiang, Y. Zhang, S. V. Dubonos, I. V. Grigorieva, and A. A. Firsov, Science 306, 666 (2004).

${ }^{14}$ Y. Zhang, Y.-W. Tan, H. L. Stormer, and P. Kim, Nature (London) 438, 201 (2005).

${ }^{15}$ C. Berger, Z. Song, X. Li, X. Wu, N. Brown, C. Naud, D. Mayou, T. Li, J. Hass, A. N. Marchenkov, E. H. Conrad, P. N. First, and W. A. de Heer, Science 312, 1191 (2006).

${ }^{16}$ S. Cahangirov, M. Topsakal, E. Akturk, H. Sahin, and S. Ciraci, Phys. Rev. Lett. 102, 236804 (2009).

${ }^{17}$ P. E. Blöchl, Phys. Rev. B 50, 17953 (1994).

${ }^{18}$ J. P. Perdew, J. A. Chevary, S. H. Vosko, K. A. Jackson, M. R. Pederson, D. J. Singh, and C. Fiolhais, Phys. Rev. B 46, 6671 (1992).

${ }^{19}$ G. Kresse and J. Hafner, Phys. Rev. B 47, 558 (1993).

${ }^{20}$ G. Kresse and J. Furthmuller, Phys. Rev. B 54, 11169 (1996).

${ }^{21}$ J. M. Soler, E. Artacho, J. D. Gale, A. Garcia, J. Junquera, P. Ordejon, and D. Sanchez-Portal, J. Phys.: Condens. Matter 14,
2745 (2002).

${ }^{22}$ D. Alfè, Comput. Phys. Commun. 180, 2622 (2009).

${ }^{23}$ M. Shishkin and G. Kresse, Phys. Rev. B 74, 035101 (2006).

${ }^{24}$ R. T. Senger, S. Tongay, E. Durgun, and S. Ciraci, Phys. Rev. B 72, 075419 (2005).

${ }^{25}$ G. Henkelman, A. Arnaldsson, and H. Jonsson, Comput. Mater. Sci. 36, 354 (2006).

${ }^{26}$ S. Tongay, E. Durgun, and S. Ciraci, Appl. Phys. Lett. 85, 6179 (2004).

${ }^{27}$ S. Tongay, R. T. Senger, S. Dag, and S. Ciraci, Phys. Rev. Lett. 93, 136404 (2004).

${ }^{28}$ D. Bimberg, R. Blachnik, M. Carolone, P. J. Dean, Th. Grave, G. Harbeke, K. Hubner, U. Kaufmann, W. Kress, O. Madelung, W. von Munch, U. Rossler, J. Schneider, M. Schulz, and M. S. Skolnick, in Zahlenwerte und Funktionen aus Naturwissenschaft und Technik, Landolt-Bornstein, New Series, edited by $\mathrm{O}$. Madelung, M. Schulz, and H. Weiss (Springer-Verlag, Berlin, 1982) Group III, Pt. 17.

${ }^{29}$ J. Serrano, J. Strempfer, M. Cardona, M. Schwoerer-Bohning, H. Requardt, M. Lorenzen, B. Stojetz, P. Pavone, and W. J. Choyke, Appl. Phys. Lett. 80, 4360 (2002).

${ }^{30}$ SiC Power Materials: Devices and Applications, edited by Zhe Chuan Feng (Springer-Verlag, Berlin, 2004).

${ }^{31}$ L. Sun, Y. Li, Z. Li, Q. Li, Z. Zhou, Z. Chen, J. Yang, and J. G. Hou, J. Chem. Phys. 129, 174114 (2008).

${ }^{32}$ H. Şahin, S. Cahangirov, Topsakal M., E. Bekaroglu, E. Akturk, R. T. Senger, and S. Ciraci, Phys. Rev. B 80, 155453 (2009).

${ }^{33}$ M. Topsakal and S. Ciraci, Phys. Rev. B 81, 024107 (2010).

${ }^{34}$ M. Topsakal, S. Cahangirov, and S. Ciraci, arXiv:0908.2887 (unpublished).

${ }^{35}$ Y.-W. Son, M. L. Cohen, and S. G. Louie, Phys. Rev. Lett. 97, 216803 (2006).

${ }^{36}$ P. Lou and J. Y. Lee, J. Phys. Chem. C 113, 12637 (2009).

${ }^{37}$ P. Lou and J. Y. Lee, J. Phys. Chem. C 113, 21213 (2009).

${ }^{38}$ P. Esquinazi, D. Spemann, R. Höhne, A. Setzer, K.-H. Han, and T. Butz, Phys. Rev. Lett. 91, 227201 (2003).

${ }^{39}$ A. Hashimoto, K. Suenaga, A. Gloter, K. Urita, and S. Iijima, Nature (London) 430, 870 (2004).

${ }^{40}$ O. V. Yazyev and L. Helm, Phys. Rev. B 75, 125408 (2007).

${ }^{41}$ M. A. H. Vozmediano, M. P. Lopez-Sancho, T. Stauber, and F. Guinea, Phys. Rev. B 72, 155121 (2005).

${ }^{42}$ L. Brey, H. A. Fertig, and S. Das Sarma, Phys. Rev. Lett. 99, 116802 (2007).

${ }^{43}$ M. Topsakal, E. Akturk, H. Sevinçli, and S. Ciraci, Phys. Rev. B 78, 235435 (2008).

${ }^{44}$ E. H. Lieb, Phys. Rev. Lett. 62, 1201 (1989). 